\title{
EL DISCURSO NACIONAL-POPULAR DE ANDRÉS MANUEL LÓPEZ OBRADOR [2018-2020] *
}

\section{The national-popular discourse of Andrés Manuel López Obrador (2018-2020)}

Noé Hernández Cortez**

Jesús Moya Vela***

Ernesto Menchaca Arredondo ${ }^{* * * *}$

Recepción: 15 de agosto de 2020. Aceptación: 12 de abril de 2021

DOI: http:/ /dx.doi.org/10.21017/Rev.Repub.2021.v31.a106

\section{RESUMEN}

El llamado giro a la izquierda, en la primera década del siglo XXI en el orbe latinoamericano, reconfiguró los discursos populistas en el mapa político de esta región. México llegó tarde a la ola populista de izquierda, pues esta se da hasta el ascenso democrático del partido MORENA y su líder Andrés Manuel López Obrador, quien llega al poder en el 2018 en un contexto en el que la derecha se consolida en Latinoamérica. A partir de un análisis de los discursos del presidente de México, Andrés Manuel López Obrador, en el período 20182020, observamos las condiciones de posibilidad de la construcción de un discurso nacional-popular, que pretende articular de forma hegemónica, una nueva forma de hacer política con un discurso de rechazo y crítica de la «élite política corrupta» y de la ideología neoliberal en México. Teniendo como marco de referencia los conceptos de lo «nacional-popular» de Gramsci (1999), y de

* Artículo de reflexión derivado del proyecto de investigación «Los partidos populistas en América Latina en el siglo XXI: los casos de MORENA en México y el PSUV en Venezuela» (UAZ-2019-37948) registrado en la Universidad Autónoma de Zacatecas.

** Doctor de Investigación en Ciencias Sociales con mención en Ciencia Política por la FLACSO, sede México. Docente investigador de tiempo completo titular «C» en la Unidad Académica de Ciencia Política de la Universidad Autónoma de Zacatecas. Responsable del proyecto UAZ-2019-37948.

*** Doctor en Ciencia Política por la Universidad Autónoma de Zacatecas. Docente investigador de tiempo completo titular «C» en la Unidad Académica de Ciencia Política de la Universidad Autónoma de Zacatecas. Colaborador en el proyecto UAZ-2019-37948.

**** Doctor en Ciencia Política por la Universidad Autónoma de Zacatecas. Docente investigador de tiempo completo titular «C» en la Unidad Académica de Ciencia Política de la Universidad Autónoma de Zacatecas. Coordinador de la Maestría en Ciencia Política de la UAZ. Colaborador en el proyecto UAZ-2019-37948. 
Portantiero y De Ípola (1981) y el «discurso populista» de Laclau (2010), presentamos un primer avance de nuestra investigación, señalando que lo nacional-popular es el rasgo distintivo del discurso populista del presidente de México, a través del cual configura su proyecto político llamado por él mismo «la Cuarta Transformación de México».

Palabras clave: populismo, discurso, nacional-popular, Andrés Manuel López Obrador.

\begin{abstract}
The so-called left turn, in the first decade of the 21st century in the Latin American area, restructured the populist discourses in the political map of this region. Mexico arrived late to the left-wing populist wave, since it happened until the democratic rise of the political party MORENA and its leader Andrés Manuel López Obrador, who takes office in 2018 in a context where the right is strengthening in Latin America. From an analysis of the discourses of the President of Mexico, Andrés Manuel López Obrador, in the period 2018-2020, we observe the conditions of possibility of the construction of a popular-national discourse, which seeks to articulate, in an hegemonic way, a new way to make politics with his discourse of rejection and criticism of the «corrupt political elite» and the neoliberal ideology in Mexico. Taking as a frame of reference the concepts of the "national-popular" of Gramsci (1986), and Portantiero and De Ípola (1981) and the "populist discourse" of Laclau (2010), we present a first advance of our research, pointing out that the national-popular is the distinctive feature of the populist discourse of the President of Mexico, through which he configures his political project named by himself "the Fourth Transformation of Mexico".
\end{abstract}

Key-words: populism, discourse, national-popular, Andrés Manuel López Obrador.

\title{
INTRODUCCIÓN
}

El llamado giro a la izquierda en América Latina en la primera década del siglo XXI es consecuencia de los movimientos nacional-populares en Venezuela, Ecuador y Bolivia, que dan inicio a una resignificación en el imaginario político latinoamericano como posibilidad de una ruta ideológica de izquierda desde el Estado, construyendo al sujeto político de «pueblo» como una realidad histórica de acción política (Cancino, 2012). El predominio del neoliberalismo desde la década de los ochenta en América Latina, agudizó la desigualdad y el descontento entre las masas populares, la articulación entre 
la élite política y económica naturalizó el discurso neoliberal desde el Estado, convirtiéndose así la ideología neoliberal en el «nuevo evangelio político» de la clase dominante y en la «tecnología de poder» para dominar los espacios de la vida política y social. El Consenso de Washington había institucionalizado el «neoliberalismo desatado» (Palma, 2018; 2009).

En este contexto, el movimiento nacional-popular encabezado por Hugo Chávez en Venezuela cristalizó en gobierno en 1989, constituyendo una coyuntura política que encauzó la lucha popular por los canales institucionales de la democracia procedimental, en ese momento en crisis por la corrupción institucionalizada y la fragmentación política del bipartidismo predominante en Venezuela. El chavismo construyó el primer discurso nacional-popular que hizo frente a la hegemonía del neoliberalismo, a la vez que apelaba al pueblo como sujeto político (Hernández, 2011). No obstante, se identifican lógicas políticas distintas en los movimientos nacional-populares en América Latina. El gobierno nacional-popular de Rafael Correa (2007-2017) en Ecuador se caracterizó por una visión tecnocrática de las políticas públicas para incluir a los excluidos del sistema político, a través de políticas redistributivas para combatir la desigualdad. No obstante, la relación política entre el gobierno de Rafael Correa y la sociedad se ejerció de forma vertical, sin una participación democrática amplia. El gobierno de Evo Morales (2006-2019) que por el origen de su partido el MAS (Movimiento al Socialismo), arraigado en las prácticas de las asambleas comunitarias democráticas en la toma de decisiones políticas, hizo posible una mayor apertura a la democratización a través de la participación política (De la Torre, 2013).

En el 2018 en México el candidato presidencial del partido Movimiento Regeneración Nacional (Morena), en una coalición electoral denominada «Juntos Haremos Historia» ganó las elecciones presidenciales, apoyado por las bases populares de su reciente partido, Morena. ${ }^{1}$ Así, en el México contemporáneo llega al poder un gobierno con un discurso nacional-popular. Al igual que el Partido Socialista Unido de Venezuela (PSUV), Morena agrupa un conjunto de actores políticos con diversos tintes ideológicos, pero que los unió estratégicamente la factibilidad de que (en el escenario político de las elecciones presidenciales del 2018 en México) su líder Andrés Manuel López obrador (AMLO) tenía grandes posibilidades de obtener el triunfo electoral. Asimismo, el apoyo político de Morena y AMLO provenía de unas activas bases populares, que se habían movilizado para promover el voto a lo largo y ancho del país.

1 Morena como partido político obtuvo su registro del Instituto Nacional Electoral (INE) el 9 de julio de 2014 . 
El presente artículo se divide en cuatro partes. En una primera parte establecemos una discusión teórica sobre las conexiones políticas entre lo nacional-popular y la construcción del discurso populista como hegemonía. Enseguida, exponemos el discurso nacional-popular de la Cuarta Transformación como una ficción política en la construcción de pueblo a través del imaginario histórico. En una tercera parte abordamos el discurso de lo nacional-popular como desarrollo, a través del discurso de los megaproyectos impulsados por el presidente Andrés Manuel López Obrador. Finalmente, presentamos las conclusiones de la investigación.

\section{PERSPECTIVAS TEÓRICAS EN TORNO A LO NACIONAL- POPULAR Y EL POPULISMO}

Nuestra perspectiva teórica tiene como punto de partida la reflexión en torno a las posibilidades de pensar lo nacional-popular como un discurso populista que interpela al «pueblo» para formar hegemonía. Para ello consideramos que el instrumental analítico pertinente es la teoría del populismo de Laclau (2010; 1986) y Laclau y Mouffe (2011). Para algunos analistas el concepto de lo nacional-popular de Gramsci es fundamental para otorgar historicidad a las categorías analíticas del populismo que ofrece, principalmente, el Laclau de la «razón populista» (Retamozo, 2018). Sin embargo, consideramos que Laclau siempre tuvo presente, en sus formulaciones teóricas sobre el populismo, la historicidad popular del peronismo en Argentina. En este contexto, el discurso populista se puede articular con la noción de lo nacional-popular, toda vez, que la concepción de discurso de Laclau implica la interpelación al pueblo, lo que hace posible la construcción del sujeto político como una subjetividad que estructura las formas simbólicas, imaginarias y mitos de la comunidad política. En otros términos, podemos decir que el líder populista de un proyecto político nacionalpopular tiene la capacidad de producir significados que interpelen al sujeto político.

El análisis crítico que hace Laclau (2010) de la psicología de las masas de Le Bon (2014) no implicó que el primero no tuviera una influencia importante de dicha visión de la sociedad, los liderazgos y las masas (en este caso, traslapado en la acepción de «pueblo»). La influencia del psicoanálisis es también importante, ya que se considera en esa corriente que el individuo logra establecer un estado psicológico colectivo a través de la identificación e introyección del líder carismático, lo que a su vez conlleva la conformación del grupo por medio de la identificación entre los integrantes, debido a que se comparten las mismas características introyectadas (Arciga, 2001). Estos referentes teóricos y epistemológicos marcaron el análisis que Laclau hizo del populismo. El discurso populista, entendido como ficción 
política, para decirlo con Morgan (2006), permite inventar al pueblo. De ahí, la importancia de considerar al discurso populista como el medio de producir significados y sentido performativo para construir lo nacionalpopular, a través del sujeto político como expresión de las subjetividades políticas subalternas.

En ese sentido, la articulación del discurso populista con lo nacional-popular abre una prometedora estrategia metodológica para observar cómo los líderes populistas interpelan al sujeto político, lo que permite identificar sus estrategias políticas para construir hegemonía, que canalice su proyecto político. Teóricamente privilegiamos el lugar de enunciación del líder populista, pues, en el contexto político de América Latina, el discurso nacional-popular va acompañado con la irrupción del líder carismático, que intenta recuperar al Estado como aparato comprometido con lo nacional-popular (Portantiero,1981). En términos filosóficos de Laclau y Mouffe (2011), el líder populista «encarna» la unidad perdida de la comunidad política, unidad perdida en la fragmentación social del mundo moderno:

En el tipo de teorización que nos interesa analizar, los elementos sobre los que operan las prácticas articulatorias fueron inicialmente especificados como fragmentos de una totalidad estructural u orgánica pérdida. A partir del siglo XVIII, la generación romántica alemana va a hacer de la experiencia de la fragmentación y de la división el punto de partida de su reflexión teórica. El colapso, a partir del siglo XVII, de la concepción del cosmos como un orden significativo dentro del cual el hombre ocupa un lugar determinado y preciso, y su reemplazo por una concepción del sujeto como autodefinido, como una entidad que mantiene relaciones de exterioridad con el resto del universo - el desencanto weberiano del mundo - da lugar en la generación romántica del Sturm un Drang a una búsqueda anhelosa de la unidad perdida, de una nueva síntesis que permita vencer la división. La visión del hombre como expresión de una totalidad integral trata de romper con todos los dualismos - cuerpo/alma, razón/sentimiento, pensamiento/sentidos - que el racionalismo había instituido a partir del siglo XVII. Es sabido que esta experiencia de la disociación era concebida por los románticos como estrictamente ligada a la diferenciación funcional y a la división de la sociedad en clases, a la creciente complejidad de un Estado burocrático que asumía relaciones de exterioridad con las otras esferas de la vida social (Laclau y Mouffe, 2011, pp. 129-130).

Ahora bien, lo nacional-popular como discurso populista de un líder carismático se puede materializar como la pretensión de hegemonía de un proyecto político, que cruza dos dimensiones: 1) como proyecto de un modelo de desarrollo y 2) como la interpelación política a las masas populares. 
Siguiendo la lectura que hace Juan Carlos Portantiero de Gramsci, son dos fases estatales de hegemonía:

lo que interesaría es analizar a las sucesivas fases estatales como diferentes modelos de hegemonía. Cada fase estatal implica, en efecto, una modificación en las relaciones que se establecen entre estado y economía (modelo de desarrollo) y entre estado y masas (modelo de hegemonía). Esta problemáti$\mathrm{ca}$, que nos saca del terreno de una teoría general del estado para acercarnos a una sociología de sus transformaciones, permite fundar el análisis político de la producción de hegemonía como una relación específica entre masas e instituciones (Portantiero,1981, 161).

De lo anterior se desprende nuestra estrategia metodológica: observar lo nacional-popular como un discurso que interpela al sujeto político, principalmente en el imaginario de las creencias y en lo simbólico, a la par que el discurso de lo nacional-popular se materializa en un modelo de desarrollo. Para Laclau, esta interpelación coadyuva a la constitución de identidades cohesionando la diversidad en un todo cuando el discurso populista demarca en su contenido un «significante vacío» que se llena subjetivamente con la creencia de la pertenencia a esa totalidad, al pueblo (Laclau, 2010).

Ambos componentes, la interpelación del sujeto político y el modelo de desarrollo, son dos mecanismos que crean las condiciones de posibilidad de hegemonizar el proyecto político del líder de un movimiento popular, creando las condiciones estructurales de un Estado comprometido con lo nacional-popular. Como proponen Portantiero y De Ípola, un discurso nacional-popular a la altura de una reforma intelectual y moral:

Es en el interior de esta problemática donde se coloca, como un derivado natural, el tema de lo «nacional-popular» y se concibe expresamente a la hegemonía como capacidad de una clase para la construcción de una «voluntad colectiva nacional popular» sostenida sobre una gran «reforma intelectual y moral» (Portantiero y De Ípola, 1981, p. 7).

Los criterios de Portantiero y De Ípola (1981) para construir el discurso de lo nacional-popular no solo radican en el líder populista, sino que se requiere de una reforma en el orden de lo intelectual y moral, en el mundo de las ideas y valores para la acción política. Esta breve discusión teórica hace posible vislumbrar la relevancia del discurso para el análisis político de lo nacional-popular, anclado en el mundo de lo simbólico, el imaginario político y en la voluntad colectiva. 


\section{EL DISCURSO NACIONAL-POPULAR DE LA CUARTA TRANS- FORMACIÓN}

El escenario político mexicano en las últimas dos décadas ha estado marcado por el ascenso de la violencia de agentes estatales y no estatales, así como las prácticas de la corrupción en la Administración Pública en los tres órdenes de gobierno: federal, estatal y municipal. Para algunos analistas, el sentido antidemocrático de los partidos políticos en México es uno de los factores que explican la corrupción de las élites políticas en México, al cerrar el paso, en las elecciones internas de los partidos políticos, para que cualquier ciudadano pueda ser votado, ya que el procedimiento de la elección de los candidatos de los partidos es determinado por la cúpula del partido, práctica política que en México se conoce popularmente como el «dedazo» (Buscaglia, 2017).

Ante la incapacidad de representación política del sistema de partidos políticos en México, surge el partido Movimiento Regeneración Nacional (Morena), que aglutinó con un discurso nacional-popular las demandas insatisfechas de las clases populares, consolidando el proyecto político de la Cuarta Transformación a través de su líder Andrés Manuel López Obrador. En el contexto de las demandas populares no satisfechas por el sistema de partidos mexicano, Morena se presenta en la elección presidencial del 2018 a través de la coalición electoral «Juntos Haremos Historia», acompañado de un discurso nacionalpopular por medio de su líder Andrés Manuel López Obrador. En esta competencia electoral por la Presidencia de México, Morena gana la elección presidencial obteniendo el 53\% de la votación efectiva del padrón electoral nacional (Monsiváis, 2018).

En la política es importante tener presente el papel que juegan los imaginarios sociales, las creencias, las ficciones para sedimentar los proyectos políticos hegemónicos. Así, los discursos populistas de lo nacional-popular tienen sus raíces en el lenguaje del sentido común, tema olvidado por cierto en las ciencias sociales en América Latina, pues se ha pasado a evaluar el discurso político, solo a un nivel normativo de acuerdo con los estándares ontológicos de la democracia liberal. En consecuencia, al discurso populista se le ha prestado poca atención como un medio para llevar a cabo un proyecto político, principalmente en los contextos sociopolíticos de América Latina. De ahí la relevancia de Gramsci $(1971,1999)$ y sus reflexiones sobre el lenguaje, el sentido común y la cultura como elementos indispensables para configurar un proyecto político hegemónico y tener la posibilidad de comprender la producción de símbolos y significados de las colectividades populares. Al respecto escribe Gramsci:

Me parece que se puede decir que «lenguaje» es esencialmente un nombre colectivo que no supone una cosa única ni en el espacio ni en el tiempo. 
Lenguaje significa también cultura y filosofía (aun cuando en el orden del sentido común) y, por lo tanto, el hecho «lenguaje» es en realidad una multiplicidad de hechos más o menos orgánicamente coherentes y coordinados. Llevando las cosas al límite se puede decir que cada ser parlante tiene su propio lenguaje, esto es, un modo propio de pensar y de sentir. La cultura, en sus distintos grados, unifica una mayor o menor cantidad de individuos en estratos numerosos, en contacto más o menos expresivo, que se comprenden en diversos grados, etc. (Gramsci, 1971, p. 31).

Así, el sentido común viene a configurar el lenguaje colectivo de una comunidad política. El discurso populista tiene que ser capaz de interpelar a esa voluntad general, hacer posible la creación de imaginarios políticos y culturales, que articule lo que está sedimentado en la colectividad popular. Por eso el líder populista apela al sentido común para llevar a cabo su proyecto político, y la aspiración política es sedimentarlo y estabilizarlo como un discurso hegemónico. Asimismo, el lenguaje de la cultura popular está cruzado por las emociones, esto es, la política son emociones que la razón instrumental, una y otra vez, ha pretendido erradicar del campo de la política. Citamos nuevamente en extenso a Gramsci sobre este punto de las emociones en la vida colectiva:

De ello se deduce la importancia que tiene el «momento cultural», incluso en la actividad práctica (colectiva): cada acto histórico sólo puede ser cumplido por el «hombre colectivo». Esto supone el logro de una unidad «cultural-social», por la cual una multiplicidad de voluntades disgregadas, con heterogeneidad de fines, se sueldan con vistas a un mismo fin, sobre la base de una misma y común concepción del mundo (general y particular, transitoriamente operante - por vía emocional - o permanente, cuya base intelectual está tan arraigada, asimilada y vivida, que puede convertirse en pasión). Si así son las cosas, revélase la importancia de la cuestión lingüística general, o sea, del logro de un mismo «clima» cultural colectivo (Gramsci, 1971, p.31).

El discurso colectivo de la Cuarta Transformación apela a las voluntades colectivas, para que se articulen y así hegemonizar un proyecto político nacionalpopular, que contrarreste el modelo neoliberal que lleva cuatro décadas operando en la política latinoamericana y en México. Andrés Manuel López Obrador apela a la historia mexicana, pues, por su experiencia política como líder de movimientos sociales a lo largo de la reciente historia política de México, sabe que esta está conformada de repertorios simbólicos que los mexicanos tienen en su sentido común, o bien, que permite crear el horizonte de un México grandioso por sus héroes de historia patria. Este discurso populista no solo es porque fragmenta el mundo político entre la élite corrupta y el pueblo (Mudde y Rovira, 2017; Hawkins y Rovira, 2017), sino que profundiza en el sentido 
común de lo nacional-popular, y estas formas discursivas de lo nacional-popular son la política, la experiencia política latinoamericana sedimentada en el sentido común de la cultura popular.

La Cuarta Transformación, en ese sentido, es la constitución de un discurso nacional-popular, que trae la historia patria a la política nacional, pues esta ha estado hegemonizada por la retórica tecnocrática del lenguaje del neoliberalismo. Como señala Laclau (2014) la retórica es el fundamento político de la sociedad, no es una retórica en el vacío, pues es el arte retórico el que hace posible construir lo social, mediada por la política y «lo político». En ese horizonte, de acuerdo con AMLO, la Cuarta Transformación es un momento histórico que pretende asentar las condiciones de posibilidad de estructurar una política que ponga al Estado en un compromiso social con los pobres, como lo sostuvo AMLO en su discurso político de su toma de posesión como presidente de México: «Por el bien de todos, primero los pobres» (LópezObrador, 2018, diciembre 1).

La Cuarta Transformación es consecuencia de tres transformaciones políticas de la historia de México: la Independencia de México (1821), la Reforma liberal de Benito Juárez (1857) y la Revolución mexicana de 1910 (Otero, 2018). La Cuarta Transformación es una ruptura con el «pasado neoliberal»y «corrupto de México». Esta narrativa de AMLO constituye su discurso, que pretende hegemonizar en el imaginario político, para llevar a cabo su proyecto político. Un discurso que tiene sus raíces en el Cardenismo de la historia de Méxi$\mathrm{co}$, considerado el primer proyecto político nacional-popular. Es importante mencionar que la Cuarta Transformación como proyecto político de izquierda en México surge, como ya mencionamos, de una contienda político-electoral en donde AMLO obtuvo el 53\% de la votación efectiva; en ese sentido, la Cuarta Transformación como proyecto político de izquierda en México ha construido un discurso nacional-popular en un marco de legitimidad democrática.

La Cuarta Transformación es un movimiento político que se opone al modelo neoliberal, caracterizado por AMLO como corrupto y facilitador financiero, a costa del Estado, de los mercados internacionales de la globalización (López Obrador, 2018, octubre 30). En el 2017 AMLO presenta sus líneas políticas y su diagnóstico sobre la política mexicana. El neoliberalismo y la corrupción son los «males» de México. Al respecto escribe AMLO:

los pregoneros del neoliberalismo utilizan muchas mentiras para tratar de justificar esa forma de saqueo disfrazada; exaltan el mito de la supremacía del mercado, sostienen que la soberanía es un concepto caduco frente a la globalidad, que el Estado no debe promover el desarrollo ni procurar la distri- 
bución del ingreso, porque si les va bien a los de arriba les irá bien a los de abajo. Pero la idea de que tarde o temprano la riqueza «goteará» hacia la base de la sociedad es un sofisma, porque la riqueza no es líquida y mucho menos contagiosa. Los propagandistas del neoliberalismo inclusive han resucitado el viejo criterio conservador porfirista según el cual «mientras haya mundo tendremos un número muy reducido de afortunados, en contraposición con la inmensa mayoría, que luchará en vano por alcanzar los favores de la fortuna»; es decir, buscan con absurdos justificar el incumplimiento de la responsabilidad social del Estado, negar el derecho a la justicia y condenar a quienes nacen pobres a morir pobres (López Obrador, 2017, pp. 8-9).

El proyecto político de la Cuarta Transformación no está exento de una oposición política, que AMLO ha identificado, para marcar una frontera política ideológica, como «conservadores». ${ }^{2}$ La formación de antagonismos es un rasgo distintivo de la política nacional-popular, específicamente en el mundo político de América Latina. Como en Venezuela, con el ascenso al poder de Hugo Chávez como presidente de Venezuela, durante el paro Cívico-Petrolero en sus inicios de gobierno se cristalizaron los antagonismos entre el chavismo y la oposición (Hernández, 2013). En el contexto mexicano, el polo antagónico de los «conservadores» no ha articulado aún un bloque hegemónico que aspire a conformar un proyecto político coherente y viable para la acción política.

\section{EL MODELO DE DESARROLLO: MEGAPROYECTOS COMO PRO- YECTO POLÍTICO NACIONAL-POPULAR}

Las facetas estatales de hegemonía como la relación de Estado y clases populares es la política que apela al sujeto político en el campo de las creencias y lo simbólico, es aquí en donde se desarrolla el discurso nacional-popular de la Cuarta Transformación de Andrés Manuel López Obrador. La faceta estatal de hegemonía como modelo de desarrollo es donde el discurso de lo nacionalpopular se materializa; en el caso de la Cuarta Transformación, es el desarrollo de los megaproyectos del Aeropuerto Internacional Felipe Ángeles, la Refinería Dos Bocas, ${ }^{3}$ el Corredor Interoceánico del Istmo de Tehuantepec y el Tren Maya, megaproyectos que materializan la faceta estatal del proyecto de desarrollo para afianzar la hegemonía por el lado de su materialización económica (López Obrador, 2020, mayo 18).

2 Para el historiador y analista político de México, Lorenzo Meyer, «conservadores» en el discurso del presidente Andrés Manuel López Obrador son «aquellos que no desean ningún cambio realmente profundo, sino ciertas adecuaciones para mantener, básicamente, el mismo arreglo en lo económico, social, cultural y político» (Molina, 2019).

3 Refinería Dos Bocas (2020), https://dosbocas.energia.gob.mx/. 
Esta parte de nuestra investigación no pretende profundizar en cada uno de los megaproyectos, no es el propósito de la investigación. Lo que tratamos es trazar en sus líneas generales los megaproyectos de infraestructura, que está llevando a cabo el proyecto político de la Cuarta Transformación, cuya característica principal, a la par de la política social enfocada a los más vulnerables, es reestablecer el compromiso con lo nacional-popular por parte del Estado. Pues el Estado había renunciado a su relación popular con los estratos sociales marginados durante el período neoliberal. No obstante, la política de desarrollo de AMLO, por medio de estos megaproyectos, no está exenta de conflictos sociales con las comunidades en donde se van a llevar a cabo estos proyectos de desarrollo de infraestructura, principalmente en las comunidades indígenas con los proyectos del Tren Maya y el Corredor Interoceánico del Istmo de Tehuantepec (Matías, 2020; Colectivo Geocomunes, 2020; APIIDTT, 2020; Hernández, 2020).

A finales de febrero de 2020 la autoridad sanitaria de México anuncia el primer caso de COVID-19, enfermedad causada por el nuevo coronavirus SARSCoV-2. ${ }^{4}$ En este contexto de la pandemia global el 5 de abril de 2020, en su informe trimestral, ${ }^{5}$ Andrés Manuel López Obrador expone en su discurso a la ciudadanía, su proyecto de desarrollo; en un contexto complicado en términos de emergencia sanitaria y economía, AMLO apela al pueblo:

Les anticipo que a pesar de los pesares saldremos adelante, porque son más nuestras fortalezas que las debilidades o flaquezas. No olvidemos que la cultura de nuestro pueblo, que no es tan intangible como suele pensarse, siempre nos ha salvado y nos ha permitido reponernos de terremotos, huracanes, inundaciones, epidemias, tiranías, malos gobiernos y de la corrupción que ha sido la más trágica y funesta de las pestes y calamidades de México (López Obrador, 2020, abril 5).

En este contexto de emergencia sanitaria, AMLO apela a las «fortalezas» del pueblo de México, a la resignificación de la «cultura del pueblo de México», para continuar con su modelo de desarrollo, que en términos de AMLO es la búsqueda del bienestar del pueblo de México. Es importante mencionar que desde el ángulo de los proyectos políticos nacional-populares en Latinoamérica el líder populista apela a la memoria colectiva, como bien observa en su investigación Estévez Rubín de Celis (2019) sobre el caso del discurso de Cristina Fernández en Argentina:

4 Coronavirus tipo 2 del síndrome respiratorio agudo grave.

5 El presidente de México, Andrés Manuel López Obrador, desde el inicio de su mandato presidencial, ofrece un Informe Trimestral a la nación para comunicar a la ciudadanía los logros de su gobierno. 
El fenómeno que esta investigación pretende explorar es la transformación y resignificación de una matriz histórico-discursiva. Con esto hacemos referencia a la posibilidad de construcción y conquista de poder político a partir del uso y resignificación de la historia/memoria como dispositivo estructurador de sentido; esto es, trayendo al presente experiencias históricas sedimentadas en la memoria colectiva, de tal forma que se evoca un pasado que es digno de recordarse y revivirse. Se trata, en suma, de la capacidad que pueda tener un actor/sujeto político de disputar el sentido que tiene en el presente el pasado (Estévez Rubín de Celis, 2019, p. 110).

Por lo tanto, las tensiones existentes entre memoria colectiva y memoria histórica (Halbwachs, 1995) son difuminadas por los discursos nacional-populistas. El Estado recrea los acontecimientos pasados, y los líderes políticos como AMLO apropian en sus discursos memorias que permiten ensalzar un pasado que cohesiona a la comunidad política como un todo: un pueblo que trasciende el tiempo presente.

En este mismo discurso del 5 de abril de 2020, Andrés Manuel López Obrador traza las líneas para impulsar sus programas sociales y su modelo de desarrollo, estableciendo como parte fundamental de su proyecto político, como eje rector de su modelo de desarrollo, los megaproyectos de infraestructura:

Sigue en marcha la construcción del aeropuerto de Santa Lucía; la rehabilitación de las refinerías y la construcción de la planta de Dos Bocas, Paraíso, Tabasco; ahora que se cayó el precio del petróleo, se aumentará la producción de gasolinas en el país; destinaremos a ese propósito 400 mil barriles diarios adicionales para no malbaratar todo el petróleo de exportación y bajar la compra de combustibles en el extranjero; Pemex dispondrá de un recurso extra de 65 mil millones de pesos, porque se le reducirá la carga fiscal; la Comisión Federal de Electricidad cuenta con financiamiento suficiente para construir las plantas termoeléctricas en la Península de Yucatán, de Baja California Sur y en otras regiones. Está asegurado, en todo el país, el abasto de energía eléctrica (López Obrador, 2020, abril 5).

A pesar de la oposición a la construcción del Corredor Transístmico en el Istmo de Tehuantepec (Ferrocarril del Istmo) y el Tren Maya, para AMLO estos dos proyectos de infraestructura detonarán el desarrollo del sureste mexicano, abandonado históricamente por la élite política y económica, y profundizado por el modelo de desarrollo neoliberal. De ahí la relevancia para AMLO de estos proyectos de desarrollo de infraestructura, que quizá serán los proyectos que mayor oposición encontrarán en los años venideros. Como apunta AMLO: 
Ya iniciamos la modernización y ampliación del Ferrocarril del Istmo y de los puertos de Salina Cruz y Coatzacoalcos. Ahí se generarán, este año, 7 mil 300 empleos. El 30 de abril se firmará el contrato de construcción del primer tramo del Tren Maya y en mayo acordaremos la construcción de cuatro tramos más. Solo dejaremos pendientes para el 2022, 485 kilómetros que construiremos con la participación de los ingenieros militares, una vez que terminen la obra del Aeropuerto General Felipe Ángeles de Santa Lucía (López Obrador, 2020, mayo 18).

Como el proyecto político nacional-popular de desarrollo del Cardenismo en México, en donde el Estado mexicano mostraba un compromiso social con las clases populares, por medio de un modelo de desarrollo de infraestructura, en el discurso de Andrés Manuel López Obrador la materialización de sus megaproyectos significa el regreso del Estado popular, desarticulado por el modelo neoliberal que predominó en México por más de treinta años. Esta división política sobre el desarrollo entre el pasado neoliberal y el actual modelo de desarrollo de compromiso social del Estado es el discurso que intenta hegemonizar Andrés Manuel López Obrador a partir de un gobierno legítimo, que ganó en las urnas con el 53\% de la votación efectiva en la elección presidencial de México de 2018.

\section{CONCLUSIONES}

El discurso nacional-popular de Andrés Manuel López Obrador apela a las clases populares, a la memoria colectiva a través de su proyecto político denominado la Cuarta Transformación. La constitución de un Estado con compromiso con lo nacional-popular tiene que articularse con un discurso populista que apele a la subjetividad de lo popular y a un modelo de desarrollo que materialice económicamente el proyecto político de la Cuarta Transformación. Como hemos mostrado, la resignificación de la historia de México, a través de la Cuarta Transformación, es un discurso que marca su línea divisoria con el neoliberalismo y la clase política corrupta. Este antagonismo anclado en un proyecto nacional-popular, en donde el Estado vuelve a ser un actor clave e intenta articularse con las masas populares, es el nuevo discurso de izquierda, que ahora ya no viene del Sur, sino del Norte de América Latina.

Como todo movimiento nacional-popular, la Cuarta Transformación de Andrés Manuel López Obrador pretende constituirse en un movimiento popular con un discurso que identifique nuevamente a la historia de México como un pasado que es digno de rescatar, para transformar política y económicamente al país. Así, el movimiento de la Cuarta Transformación abre las condiciones de posibilidad de ser un discurso populista que trascienda el actual período de 
gobierno, para sedimentar su discurso nacional-popular, que apela a las emociones y el sentido común de las masas populares. Un discurso populista que exacerbe lo nacional-popular (Svampa, 2011), para transformar democráticamente la vida política de México. Esa es la otra historia de la democratización de América Latina, que es importante narrar y resignificar desde las luchas populares de lo nacional-popular.

\section{BIBLIOGRAFÍA}

APIIDTT (2020). Asamblea de Pueblos Indígenas del Istmo Oaxaqueño en Defensa de la Tierra y el Territorio. Recuperado de https:/ / tierrayterritorio.wordpress.com/.

Arciga, S. (2001). La psicología de las multitudes. En M. González y J. Mendoza (Comp.). Significados colectivos: procesos y reflexiones teóricas. México: Tec de Monterrey.

Buscaglia, E. (2017, junio 20). Sistema electoral mexicano y poderes fácticos [Video]. Recuperado de https:/ / www.youtube.com/watch?v=r3esQPLqGSU\&t=250s.

Cancino, H. (2012). La reemergencia del discurso nacional-popular en la nueva izquierda latinoamericana: para una discusión de los movimientos-populares. Brazilian Journal of International Relations, 1(2), 237-254.

Colectivo Geocomunes (2020). Análisis General del Proyecto de Corredor Interoceánico del Istmo de Tehuantepec. Recuperado de http://geocomunes.org/Analisis_PDF/ GeoComunes_Trans\%C3\%ADstmico_22Abril2020?fbclid=IwAR0nvw_59MmbBfh QuiYShgixbfTGEgc8BQdnbc0TICDfhKPqe5BzK_yaDM.

De la Torre, C. (2013). El populismo latinoamericano. Nueva Sociedad, 247, 120-137.

Estévez Rubín de Celis, J. I. (2019). Morfologías del peronismo clásico en el discurso de Cristina Fernández (2007-2011). Perfiles Latinoamericanos, 28(55), 109-141.

Gramsci, A. (1971). El materialismo histórico y la filosofía de Benedetto Croce. Buenos Aires: Nueva Visión.

Gramsci, A. (1999). Cuadernos de la cárcel. Tomo 5. México: Era.

Gramsci, A. (1980). Notas sobre Maquiavelo, sobre la política y sobre el Estado moderno. Madrid: Nueva Visión.

Halbwachs, M. (1995). Memoria colectiva y memoria histórica. Reis: Revista Española de Investigaciones Sociológicas, 69, 209-222.

Hawkins, K. A. y Rovira Kaltwasser, C. (2017). The Ideational Approach to Populism. Latin American Research Review, 52(4), 513-528. DOI: https://doi.org/10.25222/larr.85. 
Hernández Cortez, N. (2011). El discurso ideológico de la política social en Venezuela, 1989-2010: un enfoque postestructuralista (Tesis de Doctorado, FLACSO, México). Recuperado de https:/ / repositorio.flacsoandes.edu.ec/handle/10469/3337.

Hernández Cortez, N. (2013). Venezuela en su antagonismo: las identidades formadas a partir del conflicto del Paro Cívico Petrolero de 2002-2003. En G. Zaremberg y M. A. Muñoz (Coordinadoras), Redes y Jerarquías. Participación, representación y conflicto local en América Latina, Vol. 2, (pp. 123-142). México: FLACSO.

Hernández Cortez, N. (2020). Conferencia Acción colectiva y megaproyectos de la 4T en el Istmo de Tehuantepec, Oaxaca [Video]. Recuperado dehttps:/ / www.youtube.com/ watch?time_continue $=2 \& v=k s s U P Z V K b 2 U \& f e a t u r e=e m b \_l o g o$.

Laclau, E. (1986). Política e ideología en la teoría marxista. Capitalismo, facismo, populismo. Madrid: Siglo XXI.

Laclau, E. (2010). La razón populista. Buenos Aires: Fondo de Cultura Económica.

Laclau, E. (2014). Los fundamentos retóricos de la sociedad. Buenos Aires: Fondo de Cultura Económica.

Laclau, E. y Mouffe, Ch. (2011). Hegemonía y estrategia socialista. Hacia una radicalización de la democracia. Buenos Aires: Fondo de Cultura Económica.

Le Bon, G. (2014). Psicología de las masas. Madrid: Morata.

López Obrador, A. M. (2017). 2018. La Salida. Decadencia y renacimiento de México. México: Planeta.

López Obrador, A. M. (2018, octubre 30). Pide AMLO a ciudadanos unirse contra la corrupción [Video]. Recuperado dehttps://www.youtube.com/watch?v=MSKNTHYHYrc.

López Obrador, A. M. (2020, abril 5). Discurso del Presidente Andrés Manuel López Obrador en su Informe al pueblo de México. Recuperado de https:/ / lopezobrador.org.mx / 2020/04/05/discurso-del-presidente-andres-manuel-lopez-obrador-en-su-informe-alpueblo-de-mexico/.

López Obrador, A. M. (2018, diciembre 1). «Por el bien de todos, primero los pobres». Presidente López Obrador | Toma de posesión [Video]. Recuperado de https:/ / www.youtube.com/watch?v=w3U_rEUdD7g.

López Obrador, A. M. (2020, mayo 18). Tren Maya y Aeropuerto Felipe Ángeles reactivarán economía. Conferencia presidente AMLO [Video]. Recuperado de https:// www.youtube.com/watch?time_continue=770\&v=UiJee_WLSSk\&feature=emb_logo. 
Matías, P. (2020, abril 29). Exigen la cancelación del Programa Istmo de Tehuantepec Corredor y Tren Interoceánico. Corta Mortaja. Recuperado de http:/ / cortamortaja. com.mx/el-istmo/11241-exigen-la-cancelacion-del-programa-istmo-de-tehuan tepec-corredor-y-tren-interoceanico?fbclid=IwAR2dqc7gRdvq3pkbQhcekLapmy Dv0Ye8BAZUlpC94aAbcnHUA8OAF86toI4.

Molina, G. (2019, noviembre 02). Conservadores vs. progresistas: ¿qué significa eso que tanto repite AMLO? Los expertos te lo cuentan. Cuestione. Recuperado de https://cuestione.com/detalle/mexico/conservadores-vs-progresistas-que-significa-eso-que-tanto-repite-lopez-obrador.

Monsiváis-Carrillo, A. (2018). La izquierda populista en México: ¿Amenaza o correctivo para la democracia? Working Paper. Recuperado de https://www. researchgate.net/publication/318503604_La_izquierda_populista_en_Mexico_ Amenaza_o_correctivo_para_la_democracia.

Morgan, E. (2006). La invención del pueblo: el surgimiento de la soberanía popular en Inglaterra y Estados Unidos. Buenos Aires: Siglo XXI.

Mudde, C. y Rovira Kaltwasser, C. (2017). Populism. A very short introduction. Oxford; New York, NY: Oxford University Press.

Otero, G. (2018). Morena, y la «cuarta transformación» histórica de México. Observatorio del Desarrollo, 7(21), 37-43.

Palma, J. G. (2009). The revenge of the market on the rentiers. Why neo-liberal reports of the end of history turned out to be premature. Cambridge Journal of Economics, 33, 829-869. https://doi.org/10.1093/cje/bep037.

Palma, J. G. (2018). Por qué la economía ortodoxa transfirió su obsesión por un concepto (mercado) a la de un ritual (matemáticas). Estudios Nueva Economía, 5(1), 7-20.

Portantiero, J. C. (1981). Los usos de Gramsci. México: Folios Ediciones.

Portantiero, J. C. y De Ípola, E. (1981). Lo nacional popular y los populismos realmente existentes. Nueva Sociedad, 54, 7-18.

Refinería Dos Bocas (2020). Recuperado Junio 16, 2020, de https://dosbocas. energia.gob.mx/.

Retamozo, M. (2018). Posmarxismo: entre el populismo y lo nacional popular en América Latina. Religación. Revista de Ciencias Sociales y Humanidades, 3(12), 16-40.

Svampa, M. (2011). Argentina, una década después. Del «que se vayan todos» a la exacerbación de lo nacional-popular. Nueva Sociedad, 235, 17-34. 